\title{
Prognostic Value of Pretreated Blood Inflammatory Markers in Patients with Bone Sarcoma: A Meta-Analysis
}

\author{
Min Jiang $\mathbb{D}^{1}$, Shaowei Ma $\mathbb{D}^{2}{ }^{2}$ Zhongyan Hua $\mathbb{D}^{3},{ }^{3}$ Zhiying Zhao $\mathbb{D}^{4},{ }^{4}$ and Song Gao $\mathbb{D}^{1}$ \\ ${ }^{1}$ Department of Oncology, Shengjing Hospital of China Medical University, Shenyang 110004, China \\ ${ }^{2}$ Department of Cardiology, Shengjing Hospital of China Medical University, Shenyang 110004, China \\ ${ }^{3}$ Medical Research Center, Liaoning Key Laboratory of Research and Application of Animal Models for Environmental and \\ Metabolic Diseases, Shengjing Hospital of China Medical University, Shenyang 110004, China \\ ${ }^{4}$ Department of Clinical Epidemiology, Shengjing Hospital of China Medical University, Shenyang 110004, China \\ Correspondence should be addressed to Song Gao; gaogao0229@hotmail.com
}

Received 9 September 2020; Revised 6 March 2021; Accepted 25 March 2021; Published 9 April 2021

Academic Editor: Heng Zhou

Copyright (c) 2021 Min Jiang et al. This is an open access article distributed under the Creative Commons Attribution License, which permits unrestricted use, distribution, and reproduction in any medium, provided the original work is properly cited.

\begin{abstract}
Backgroud. Multiple studies have reported blood inflammatory markers as reliable prognostic factors in various malignant tumors. However, their prognostic effect in patients with bone sarcomas has not been determined. We performed this meta-analysis to evaluate the prognostic value of pretreated blood inflammatory markers in patients with bone sarcoma. Method. We conducted a detailed literature search in Medline and Embase databases and collected relevant publications written in English before April 2020. Overall survival (OS) and disease-free survival (DFS) were the primary and secondary outcomes, respectively. Basic features of patients, hazard ratios (HRs), and 95\% confidence intervals (CI) were retrieved to assess the correlation between pretreated blood inflammatory markers and patients with bone sarcoma. This meta-analysis used Stata 12.0. Results. A total of 10 studies containing 1845 cases were included for analysis. Nine of them evaluated the neutrophil lymphocyte ratio (NLR), 7 the platelet lymphocyte ratio (PLR), and 4 the lymphocyte monocyte ratio (LMR). Pooled results revealed that higher pretreatment NLR was associated with poorer OS (HR $=1.76,95 \% \mathrm{CI}: 1.29-2.41$, and $P<0.001)$ and $\mathrm{DFS}(\mathrm{HR}=1.77,95 \% \mathrm{CI}$ : $1.09-2.88$, and $P=0.021$ ). In contrast, a lower LMR was related to worse OS (HR $=0.73,95 \% \mathrm{CI}: 0.57-0.92$, and $P=0.009$ ), but not DFS (HR $=0.68,95 \% \mathrm{CI}: 0.41-1.11$, and $P>0.05$ ). Combined results did not show a significant predictive effect of PLR on the clinical outcomes of patients with bone sarcoma (OS : HR $=1.32,95 \%$ CI: $0.99-1.75$, and $P>0.05$; DFS: HR $=1.12,95 \%$ CI: $0.87-1.44, P>0.05)$. Conclusion. NLR and LMR might be promising predictive biomarkers for patients with bone sarcoma and could be used to stratify patients and provide personalized therapeutic strategies.
\end{abstract}

\section{Introduction}

Sarcoma refers to malignancies originating from mesenchymal tissues and can be classified as bone or soft tissue sarcoma [1]. Bone sarcoma is a rare group of tumors, mainly consisted of osteosarcoma, Ewing sarcoma, and chondrosarcoma [2]. Osteosarcoma and Ewing sarcoma share similar clinical epidemiological characteristics, primarily affecting children and adolescents $[3,4]$. The incidence rate of chondrosarcoma has been shown to exhibit a gradual increase with age [4]. Although the incidence of bone sarcoma among the whole population is known to be relatively low (less than $0.2 \%$ of all new cancers), it accounts for $6 \%$ of all childhood cancers, presenting a high rate of fatality and overall disease burden [4]. Treatment of bone sarcomas in clinical practice has been challenging. Despite combined modality treatment protocols consisting of surgery, chemotherapy, and radiation, the outcomes of patients have not significantly improved for decades. Relapse rates have been reported to remain high at about $35 \%$ [5], with the 5-year survival rate for cases with metastasis being as low as 10-30\% [5, 6]. Prognosis prejudgment is essential for clinical decision-making. However, effective prognostic biomarkers for patients with bone sarcoma are still lacking. Therefore, identifying novel parameters to effectively predict prognosis and to help clinicians with the treatment option is of grave importance. 
Accumulating evidence has revealed that cancerassociated systematic inflammation might play crucial roles in the genesis and progression of tumors [7-10]. The inflammatory response is known to be reflected by many blood biomarkers, including neutrophil lymphocyte ratio (NLR), platelet lymphocyte ratio (PLR), and lymphocyte monocyte ratio (LMR). These biomarkers can be easily calculated with a routine blood test, which is both convenient and economic. Recently, several retrospective studies have reported the prognostic value of these inflammatory biomarkers for patients with bone sarcoma, but no consensus has been reached so far. Therefore, this study is aimed at investigating the role of pretreatment blood inflammatory biomarkers on the prognosis of patients with bone sarcoma.

\section{Materials and Methods}

2.1. Search Strategy. The search was based on the Preferred Reporting Items for Systematic Reviews and Meta-Analyses (PRISMA) guidelines. We conducted a systematic electronic search of the Medline and Embase databases up to April 2020. Different combinations of keywords used for preliminary search were as follows: "neutrophil" or "neutrophils," "lymphocyte" or "lymphocytes," "platelet" or "platelets," "monocyte" or "monocytes," "osteosarcoma" or "Ewing sarcoma" or "chondrosarcoma" or "bone sarcoma," and "prognostic" or "prognosis" or "outcome" or "survival." As this was a meta-analytic study using data only from published studies, ethical approval was waived.

2.2. Inclusion and Exclusion Criteria. Inclusion criteria are listed as follows: (1) pathological diagnosis of bone sarcoma; (2) studies assessing the association of NLR or PLR or LMR or their combinations with overall survival (OS), cancerspecific survival (CSS), or recurrence-free survival (RFS); (3) accessible hazard ratio (HR) and related 95\% confidence interval (CI); (4) studies published in English; and (5) human studies. Exclusion criteria were as follows: (1) comments, reviews, case reports, conference abstracts, letters, and editorials; (2) studies containing subjects with soft tissue sarcomas; (3) studies with sample size smaller than 30; (4) overlapping or duplicate studies; (5) irrelevant studies; and (6) studies not in English.

2.3. Data Extraction and Quality Assessment. Two investigators ( $\mathrm{ZH}$ and $\mathrm{SM})$ reviewed the titles and abstracts of the articles identified in the initial search. Any discrepancy would be discussed, and a third reviewer (SG) would join in to reach consensus. The information extracted was as follows: name of first author, publication year, country, number of patients, presence or absence of metastatic patients, cutoff value, biomarker, survival outcomes, histology types, and analysis method. The HR and 95\% CI values were preferentially collected from multivariate analysis; if no relevant data were offered, univariate analysis was considered as the alternative. Two investigators assessed the quality of preliminary screening articles according to the Newcastle-Ottawa scale (NOS). Studies with NOS scores $\geq 6$ were considered high-quality and included in this meta-analysis [11].
2.4. Statistical Analysis. Considering their similarity, we combined event-free (EFS), progression-free (PFS), and diseasefree (DFS) survival as DFS. Hazard ratios and 95\% CI were applied to estimate the correlation of blood inflammatory markers and survival. The heterogeneity among studies was assessed by means of $Q$-test and $I^{2}$ of the chi-square test. If significant heterogeneity $\left(P<0.05\right.$ and $\left.I^{2}>50 \%\right)$ was observed, the random effect model was used; otherwise, the fixed effect model was employed. To identify the sources of heterogeneity, we performed subgroup analysis by tumor stage, analysis method, histological type, and ethnicity. Publication bias was conducted by means of the Begg test (funnel plots). The Stata software (Stata corporation, version 12.0, College Station, TX, USA) was used for the analysis of data, and statistical significance was considered for $P$ values $<0.05$.

\section{Results}

3.1. Search Results and Characteristics of the Included Studies. A total of 413 articles were retrieved by our initial search of the Medline and Embase databases using our search strategy. After removing duplicates, 273 articles were left to be screened. Then, 250 articles were excluded by initial review, and only 23 were further assessed for eligibility. Among them, 6 reports were excluded because they were conference abstracts, whereas another 6 did not provide sufficient data to calculate the HR of patients with bone sarcoma and 1 article with only 23 samples. At last, 10 studies were included for quantitative synthesis [12-21]. The flow chart of the literature selection process is shown in Figure 1. As the study by Vasquez et al. [16] included both osteosarcoma and Ewing sarcoma cohorts, separately reporting the HR and $95 \%$ values, we marked the study of the Ewing sarcoma cohort as Vasquez et al.-EW. Similarly, the group of Li et al. reported the effect of inflammatory markers on the prognosis of patients with osteosarcoma and Ewing sarcoma in 2 articles of the same year $[15,17]$; accordingly, we marked the Ewing sarcoma cohort as Li et al.-EW.

The publication time of 10 studies ranged from 2015 to 2020. Eight studies were from China, whereas the other 2 were from Peru and Denmark, respectively. The cutoff values of inflammatory markers were as follows: 2 to 5.3 for NLR, 118 to 200 for PLR, and 3.43 to 4.73 for LMR. The NOS score values ranged from 6 to 8 . Detailed characteristics and quality assessment of eligible studies are shown in Table 1.

\subsection{Meta-Analysis Results}

3.2.1. Correlation between Neutrophil Lymphocyte Ratio and Survival Outcomes in Bone Sarcomas. A total of 1518 patients with bone sarcomas were included in 9 studies investigating the prognostic value of NLR. All studies reported the relationship between NLR and OS. Among them, 3 studies provided the DFS, as well. As these studies were characterized by significant heterogeneity among them (OS: $P<0.05$ and $I^{2}=78.2 \%$; DFS: $P=0.059$ and $I^{2}=64.7 \%$ ), the random effect model was used to analyze both OS and DFS. These results revealed that the elevated NLR was significantly correlated with poorer $\mathrm{OS}(\mathrm{HR}=1.76,95 \% \mathrm{CI}$ : $1.29-2.41$, and 


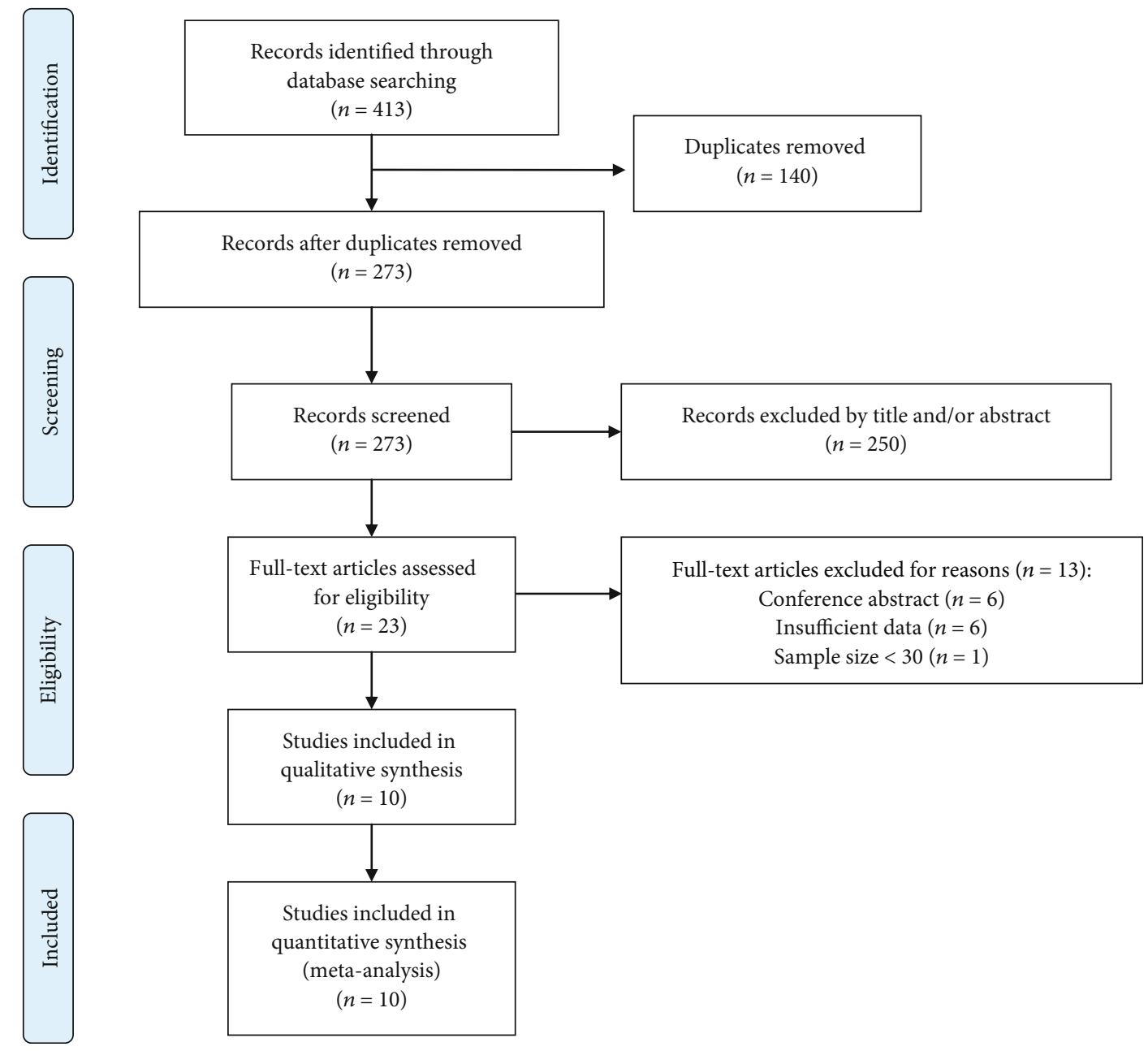

FIGURE 1: PRISMA flowchart of record search and selection.

TABLE 1: Basic characteristic of included study.

\begin{tabular}{|c|c|c|c|c|c|c|c|c|c|c|}
\hline Author & Year & Country & $\begin{array}{c}\text { Sample } \\
\text { size }\end{array}$ & Stage & $\begin{array}{l}\text { Cutoff } \\
\text { value }\end{array}$ & Markers & Outcome & $\begin{array}{c}\text { Histology } \\
\text { type }\end{array}$ & $\begin{array}{c}\text { Analysis } \\
\text { method }\end{array}$ & $\begin{array}{l}\text { NOS } \\
\text { score }\end{array}$ \\
\hline Yang et al. & 2020 & China & 133 & Mixed & $2.96 ; 4.44$ & NLR, LMR & OS, PFS & Osteosarcoma & MA & 8 \\
\hline $\mathrm{Xu}$ et al. & 2019 & China & 150 & Mixed & $2.7 ; 200$ & NLR, PLR & OS, DFS & Chondrosarcoma & MA & 8 \\
\hline Huang et al. & 2019 & China & 126 & Mixed & $2.1 ; 163.2$ & NLR, PLR & OS & Osteosarcoma & MA & 7 \\
\hline Li et al. & 2017 & China & 122 & Mixed & $\begin{array}{c}2.38 ; 131 \\
4.41\end{array}$ & $\begin{array}{l}\text { NLR, PLR } \\
\text { LMR }\end{array}$ & OS & Ewing sarcoma & UA & 7 \\
\hline Vasquez et al. & 2017 & Peru & 78 & Mixed & $2 ; 150$ & NLR, PLR & OS & $\begin{array}{l}\text { Osteosarcoma; } \\
\text { Ewing sarcoma }\end{array}$ & UA; MA & 6 \\
\hline Li et al. & 2017 & China & 216 & Mixed & $2.65 ; 118$ & NLR, PLR & OS & Osteosarcoma & UA & 7 \\
\hline Xia et al. & 2016 & China & 359 & Mixed & $3.43 ; 122$ & NLR, PLR & OS, PFS & Osteosarcoma & MA & 7 \\
\hline Liu et al. & 2016 & China & 162 & Mixed & $\begin{array}{c}2.57 ; 123.5 \\
4.73\end{array}$ & $\begin{array}{l}\text { NLR, PLR } \\
\text { LMR }\end{array}$ & OS & Osteosarcoma & MA & 7 \\
\hline Ninna et al. & 2016 & Denmark & 172 & Nonmetastatic & 5.3 & NLR & OS & $\begin{array}{c}\text { Osteosarcoma } \\
\text { Ewing sarcoma } \\
\text { Chondrosarcoma }\end{array}$ & MA & 8 \\
\hline Liu et al. & 2015 & China & 327 & Mixed & 3.43 & LMR & OS, EFS & Osteosarcoma & MA & 8 \\
\hline
\end{tabular}




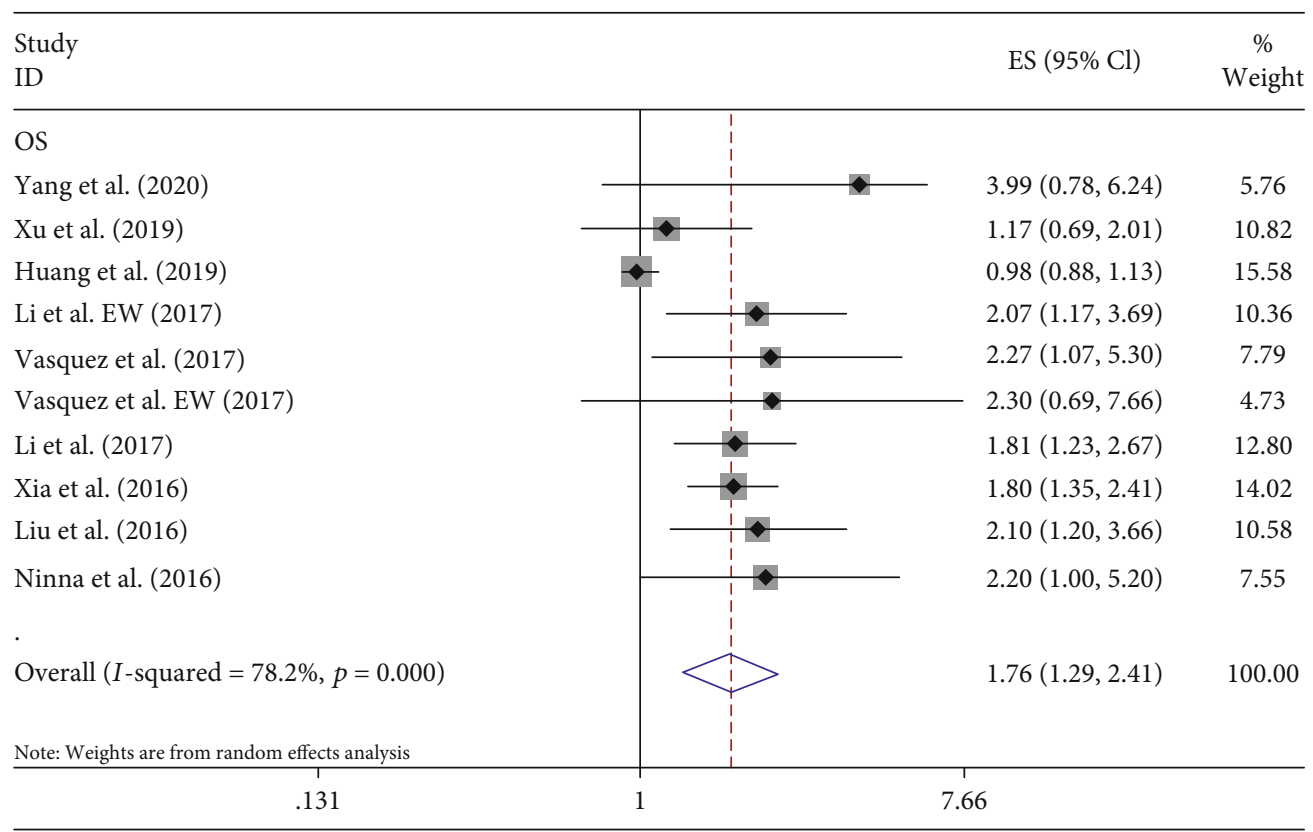

(a)

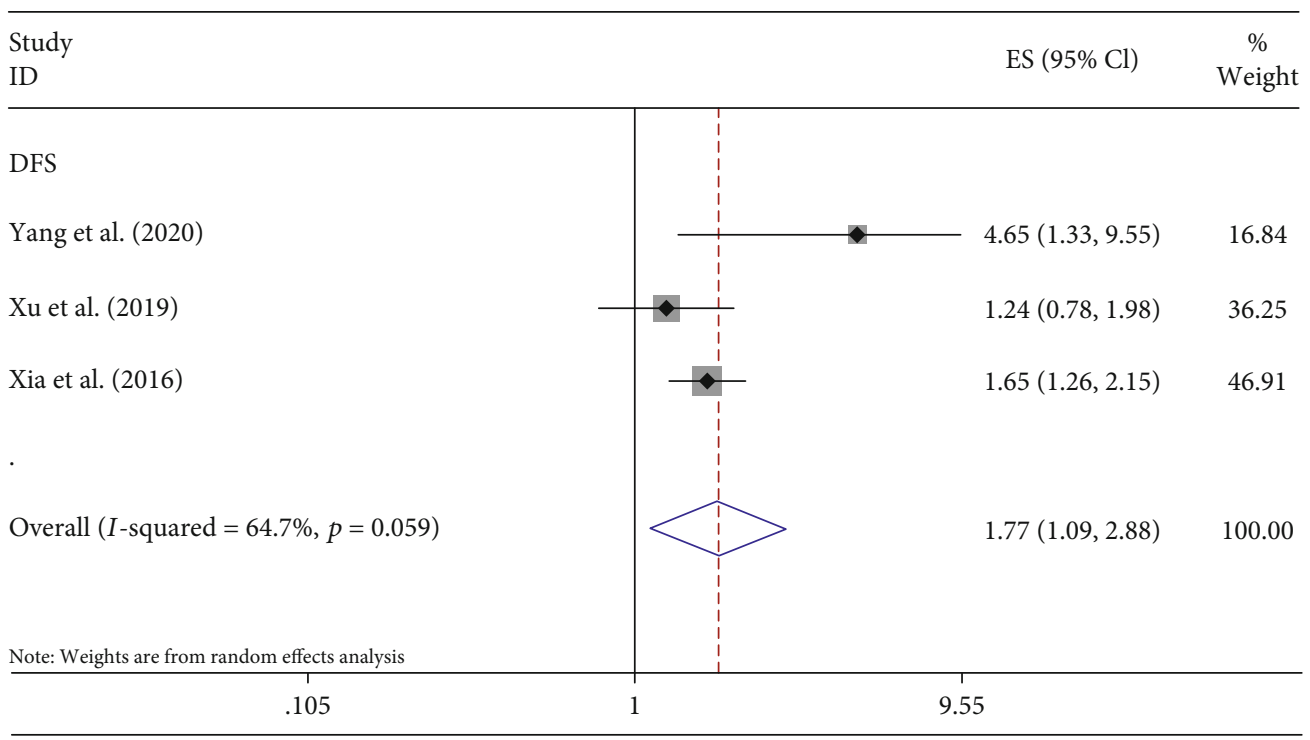

(b)

Figure 2: Forest plots of the prognostic effect of NLR for (a) OS and (b) DFS.

$P<0.001)$ (Figure $2(\mathrm{a}))$ and poorer DFS $(\mathrm{HR}=1.77,95 \% \mathrm{CI}$ : $1.09-2.88$, and $P=0.021$ ) (Figure 2(b)).

Subgroup analysis illustrated that NLR was associated with poor OS in most conditions, except for chondrosarcoma and studies not including metastatic cases. This predictive connection was not observed to be affected by either ethnicity or the method of analysis. Details are shown in Table 2.

3.2.2. Correlation between Platelet Lymphocyte Ratio and Survival Outcomes in Bone Sarcomas. Seven studies of 1213 patients with bone sarcoma reported the prognostic role of PLR for OS, with 2 of them reporting DFS. We noted a significant heterogeneity among studies for OS $(P<0.05$ and
$\left.I^{2}=69.6 \%\right)$, but no significant heterogeneity for DFS $\left(P>0.05\right.$ and $\left.I^{2}=0\right)$. The random and fixed effect models were used for OS and DFS analyses, respectively. Results showed that the increase of PLR was not significantly related with either lower OS (HR $=1.32$, 95\% CI: 0.99-1.75, and $P$ $>0.05$ ) (Figure $3(\mathrm{a})$ ) or lower DFS $(\mathrm{HR}=1.12,95 \% \mathrm{CI}$ : $0.87-1.44$, and $P>0.05$ ) (Figure 3(b)).

Although pooled results showed no correlation between PLR and OS in patients with bone sarcoma, subgroup analysis revealed that elevated PLR was correlated with poor OS in patients with Ewing sarcoma, non-Asian patients, and studies analyzed by the univariate method. Details are shown in Table 3. 
TABLE 2: Subgroup analysis of the prognostic value of NLR for OS.

\begin{tabular}{|c|c|c|c|c|c|}
\hline Subgroup analysis & No. of studies & $I^{2}(\%)$ & HR & $95 \% \mathrm{CI}$ & $P$ \\
\hline $\begin{array}{l}\text { OS } \\
\text { Total }\end{array}$ & 9 & 78.2 & 1.76 & $1.29-2.41$ & $<0.001$ \\
\hline \multicolumn{6}{|l|}{ Histology type } \\
\hline Osteosarcoma & $6^{*}$ & 85.4 & 1.78 & $1.18-2.69$ & 0.006 \\
\hline Ewing sarcoma & 2 & 0 & 2.11 & $1.26-3.54$ & 0.005 \\
\hline Chondrosarcoma & 1 & - & 1.17 & $0.69-2.01$ & 0.557 \\
\hline Mixed & 1 & - & 2.2 & $0.96-5.02$ & 0.061 \\
\hline \multicolumn{6}{|l|}{ Ethnicity } \\
\hline Asian & 7 & 83.1 & 1.66 & $1.17-2.36$ & 0.005 \\
\hline Non-Asian & 2 & 0 & 2.25 & $1.34-3.77$ & 0.002 \\
\hline \multicolumn{6}{|l|}{ Analysis method } \\
\hline MA & $7^{+}$ & 81 & 1.7 & $1.16-2.49$ & 0.006 \\
\hline UA & 3 & 0 & 1.76 & $1.29-2.41$ & $<0.001$ \\
\hline \multicolumn{6}{|l|}{ Metastasis status } \\
\hline With metastatic cases & 8 & 79.6 & 1.73 & $1.25-2.4$ & 0.001 \\
\hline Without metastatic cases & 1 & - & 2.2 & $0.96-5.02$ & 0.061 \\
\hline
\end{tabular}

*Vasquez et al. study reported Ewing sarcoma cohort and osteosarcoma cohort separately. +: Vasquez et al. study reported NLR of Ewing sarcoma cohort by means of MA and osteosarcoma cohort with UA. Abbreviations: NLR: neutrophil lymphocyte ratio; HR: hazard ratio; CI: confidence interval; MA: multivariate analysis; UA: univariate analysis.

\subsubsection{Correlation between Lymphocyte Monocyte Ratio and} Survival Outcomes in Bone Sarcomas. Four studies of 744 patients with bone sarcoma studied the prognostic effect of LMR on OS, with 2 of them reporting DFS. The fixed effect model showed that the decrease of LMR was associated with poor OS (HR $=0.73,95 \% \mathrm{CI}: 0.57-0.92$, and $P=0.009)$, not revealing significant heterogeneity $\left(P>0.05\right.$ and $\left.I^{2}=0\right)$ (Figure 4(a)). In contrast, we observed significant heterogeneity between studies for DFS $\left(P<0.05\right.$ and $\left.I^{2}=76.1 \%\right)$. Analysis with the random effect model showed that the level of LMR was not related with DFS (HR $=0.68,95 \% \mathrm{CI}: 0.41-1.11$, and $P>0.05$ ) of patients with bone sarcoma (Figure $4(\mathrm{~b})$ ).

3.3. Publication Bias. The funnel plots presented in Figure 5 revealed a slight publication bias of NLR for OS (Egger's test: $P=0.002$; Begg's test: $P=0.721)$, but no publication bias of NLR for DFS (Egger's test: $P=0.615$; Begg's test: $P=1.0$ ). We subsequently calculated the new HR values using trim and fill methods (HR: 1.647; 95\% CI: 1.232-2.201; $P=0.001$; random effects), which further indicated the prognostic value of NLR for OS. There was no evidence of publication bias in the meta-analysis of the PLR value and OS. The Begg's and Egger's $P$ values for PLR were 0.584 and 0.067 , respectively. No potential publication bias was observed in the prognostic value of LMR and OS, as well (Egger's test: $P=0.657$; Begg's test: $P=0.734)$.

\section{Discussion}

The Enneking and TNM staging systems have been serving as the foundation for predicting the prognosis of patients and determining proper treatment in bone sarcomas for a long time. However, these staging systems are known to only focus on the features of the tumor itself, such as its biological behavior, location, and size, without treating the malignant tumor as a systematic disease. As patients of the same tumor stage might vary in their clinical outcomes, current staging systems are not enough to precisely predict the prognosis of patients with bone sarcoma. Exploring laboratory parameters as potential prognostic markers might be a good strategy in complementing the existing staging system for improved stratification of patients.

The systemic inflammatory response has been suggested to have a crucial role in the development and progression of malignancies. Despite advanced progress in understanding the association between inflammatory biomarkers, such as NLR, PLR, LMR, and the prognosis of various cancers [2225 ], the impact of inflammatory markers on the clinical prognosis of patients with bone sarcoma remains obscure. To our knowledge, this is the first attempt to appraise the correlation between NLR, PLR, LMR, and prognosis of patients with bone sarcoma. In our study, 3 major histological subtypes were included: osteosarcoma, Ewing sarcoma, and chondrosarcoma. Pooled results indicated that both NLR and LMR, but not PLR, were associated with the survival of patients with bone sarcoma. Our results showed that elevated pretreated NLR had an unfavorable influence on both OS and DFS in bone sarcomas. On the other hand, the decreased level of LMR was shown to be associated with poorer OS without any exhibited heterogeneity. The prognostic value of NLR for OS was not observed to be attenuated by subgroup analysis of NLR for OS based on ethnicity, histology types, and analytical method. Taken all these into consideration, our results suggested that NLR and LMR might serve as helpful prognostic markers in bone sarcomas.

The inflammatory response could cause neutrophilia, thrombocytosis, and lymphopenia [26]. Neutrophils have been considered as the major source of the vascular 


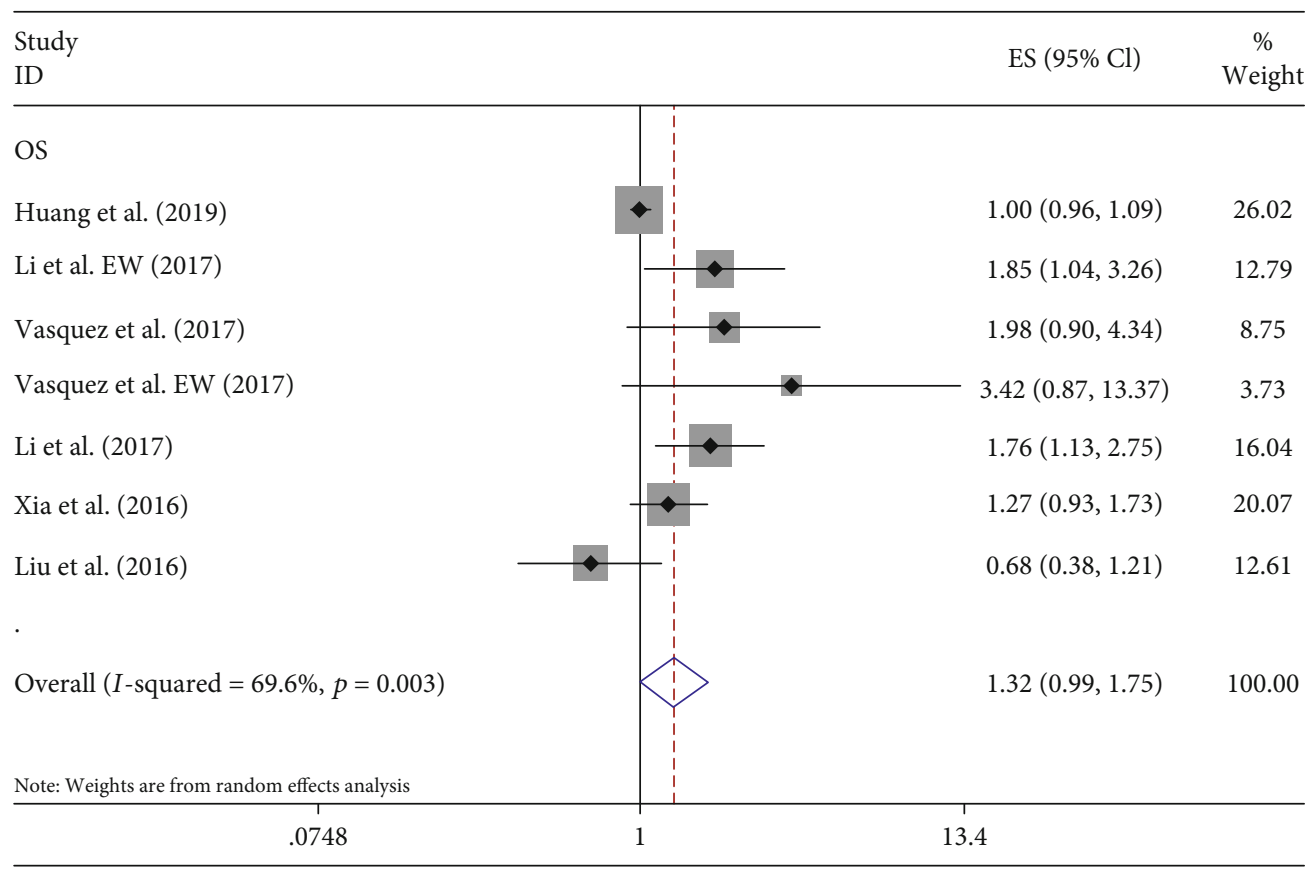

(a)

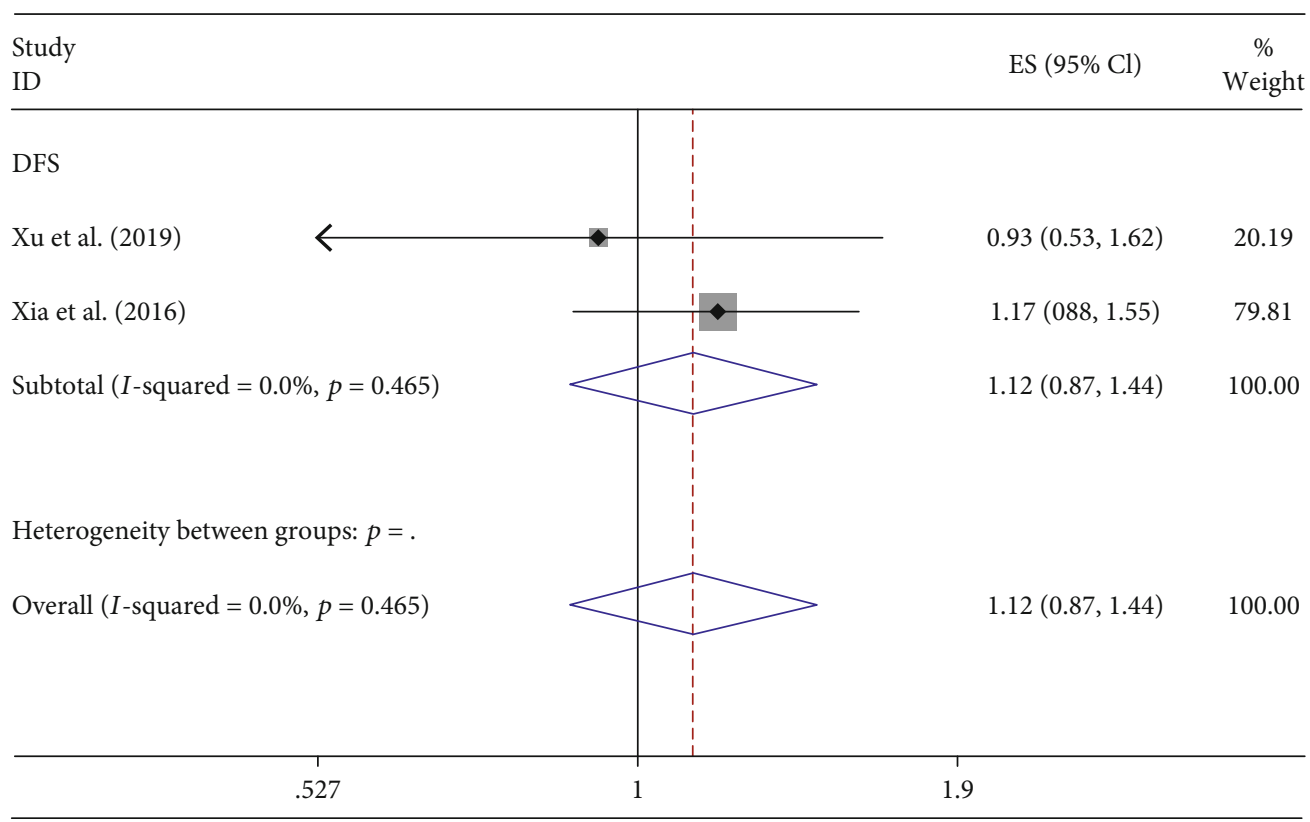

(b)

FIgURE 3: Forest plots of the prognostic effect of PLR for (a) OS and (b) DFS.

endothelial growth factor (VEGF), which is known to be a critical factor in tumor angiogenesis [27]. Besides VEGF, neutrophils are known to secrete other tumor-promoting factors, including hepatocyte growth factor [28], interleukin-8 (IL-8), IL-6 [29], and tumor necrosis factor [29], creating a favorable microenvironment for tumor survival. These neutrophil-induced inflammatory cytokines have been also demonstrated to help tumor cells subvert immune surveillance [30]. Elevated levels of platelets have been reported to not only accelerate angiogenesis of tumor and prevent cytolysis [31, 32 ], but also act as a chemoattractant in promoting the migra- tion of cancer cells [33]. Tumor-associated macrophages, mainly originating from monocytes, have also been shown to induce angiogenesis, metastasis, and immune-suppression of tumor $[34,35]$, whereas lymphocyte-dependent cellular immune response is known to be of great importance in the immunological destruction of cancer cells [36]. Elevated lymphocyte infiltration in the tumor site has been suggested to be associated with favorable outcomes $[37,38]$. In contrast, lymphopenia implied the impairment of the host immune response to tumor and was reported to be correlated with the severity of diseases $[39,40]$. Because of all this background 
TABLE 3: Subgroup analysis of the prognostic value of PLR for OS.

\begin{tabular}{|c|c|c|c|c|c|}
\hline Subgroup analysis & No. of studies & $I^{2}(\%)$ & HR & $95 \% \mathrm{CI}$ & $P$ value \\
\hline $\begin{array}{l}\text { OS } \\
\text { Total }\end{array}$ & 7 & 69.6 & 1.32 & $0.99-1.75$ & 0.059 \\
\hline \multicolumn{6}{|l|}{ Histology type } \\
\hline Osteosarcoma & 5 & 68.4 & 1.19 & $0.9-1.57$ & 0.229 \\
\hline Ewing sarcoma & 2 & 0 & 2.03 & $1.2-3.43$ & 0.009 \\
\hline \multicolumn{6}{|l|}{ Ethnicity } \\
\hline Asian & 5 & 71.6 & 1.21 & $0.91-1.60$ & 0.19 \\
\hline Non-Asian & 2 & 0 & 2.27 & $1.15-4.49$ & 0.019 \\
\hline \multicolumn{6}{|l|}{ Analysis method } \\
\hline MA & 4 & 56 & 1.08 & $0.83-1.39$ & 0.569 \\
\hline UA & 3 & 0 & 1.87 & $1.33-2.62$ & $<0.001$ \\
\hline
\end{tabular}

Abbreviations: PLR: platelet lymphocyte ratio; HR: hazard ratio; CI: confidence interval; MA: multivariate analysis; UA: univariate analysis.

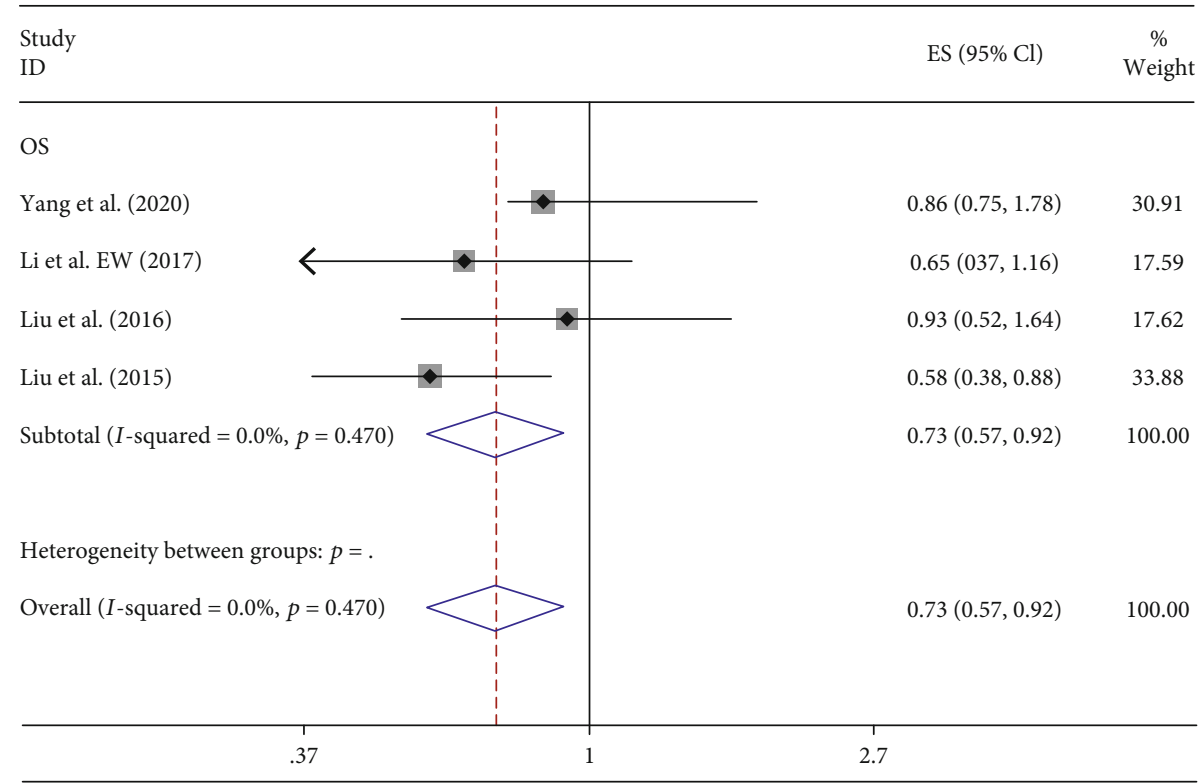

(a)

\begin{tabular}{|c|c|c|}
\hline $\begin{array}{l}\text { Study } \\
\text { ID }\end{array}$ & ES $(95 \% \mathrm{Cl})$ & $\begin{array}{c}\% \\
\text { Weight }\end{array}$ \\
\hline \multicolumn{3}{|l|}{ DFS } \\
\hline \multicolumn{3}{|l|}{ Yang et al. (2020) } \\
\hline Liu et al. (2015) & $0.53(0.39,0.76)$ & 50.56 \\
\hline Overall $(I$-squared $=76.1 \%, p=0.041)$ & $0.68(0.41,1.11)$ & 100.00 \\
\hline \multicolumn{3}{|l|}{ Note: Weights are from random effects analysis } \\
\hline .389 & 2.57 & \\
\hline
\end{tabular}

(b)

Figure 4: Forest plots of the prognostic effect of LMR for (a) OS and (b) DFS. 


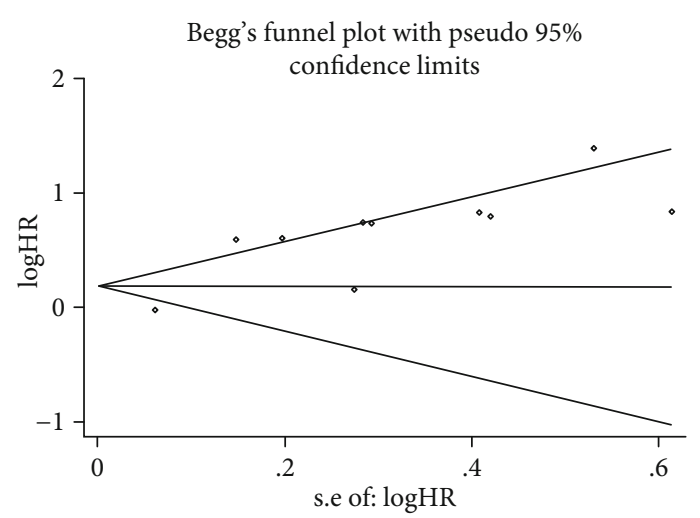

(a)

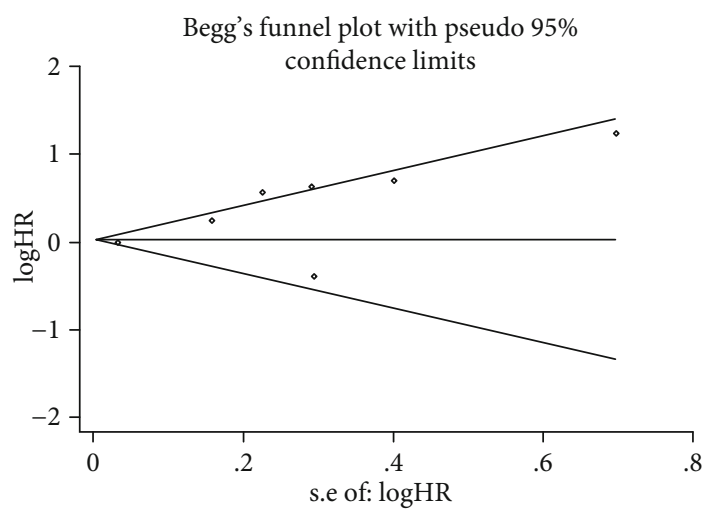

(c)

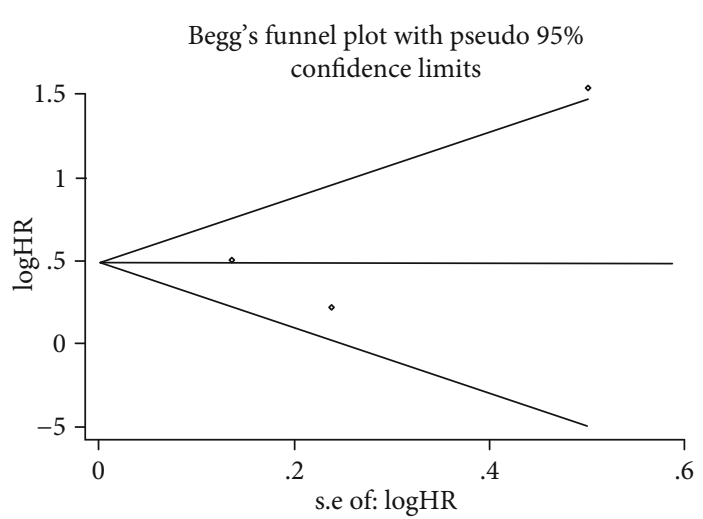

(b)

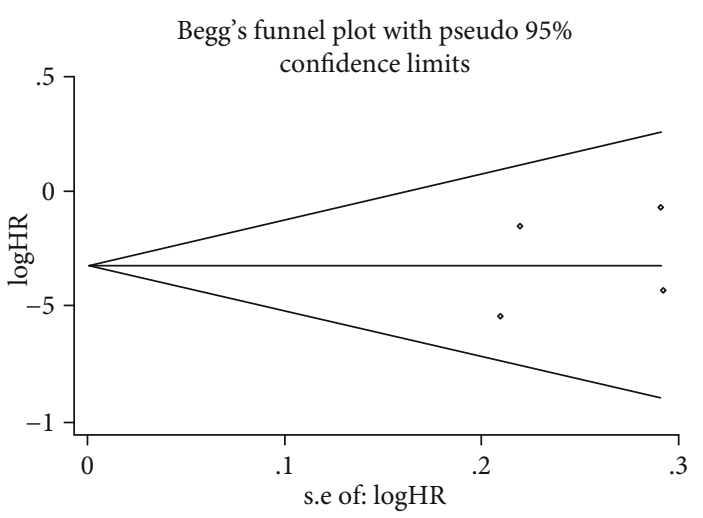

(d)

Figure 5: Analysis of publication bias for the relationship between inflammatory markers and prognosis in bone sarcoma. (a) Begg's funnel plot for NLR and OS. (b) Begg's funnel plot for NLR and DFS. (c) Begg's funnel plot for PLR and OS. (d) Begg's funnel plot for LMR and OS.

of the inflammatory response, inflammation-based prognostic indicators have emerged in the clinical management of patients with cancer.

Due to the low incidence, few studies are specifically aimed at exploring the prognostic value of inflammatory markers in bone sarcomas. Previous studies and metaanalyses have reported the prognostic role of different inflammatory markers in soft tissue sarcomas (STSs) [41, 42]. Elevated NLR and PLR and reduced LMR were demonstrated to be correlated with poor clinical outcomes in patients with STS $[43,44]$. However, most studies have analyzed STSs together with osteoblastic tumors. Considering their difference, we separated osteoblastic tumors from STSs in this meta-analysis and got consistent results with previous studies. Although no statistical significance was observed, PLR still tended to predict poor OS with a $P$ value of 0.059 .

Several studies have indicated that the C-reactive protein (CRP) and the Glasgow prognostic score were also important inflammatory prognostic indicators $[15,45]$. However, CRP was not a routine examination as part of the pretreatment assessment of patients with bone sarcoma in many hospitals. In comparison, NLR, PLR, and LMR were easy to be obtained just by performing a routine blood test. In recent years, noncoding RNAs have also been reported to be associated with the clinical prognosis of patients with bone sarcoma [46, 47].

However, the higher cost for the detection of noncoding RNAs has limited its general application in clinical practice.
The inflammatory markers in our study possess the advantage of low cost and easy accessibility, which could be suitable for routine monitoring in predicting the clinical outcome in patients with bone sarcomas.

Despite all the advantages, the values of NLR, PLR, and LMR would be altered by certain diseases (such as infections, cardiac events, atherosclerosis, and abnormal thyroid function) and drugs like nonsteroidal anti-inflammatory drugs (NSAIDS). Although all the enrolled studies have claimed to exclude patients with diseases/drugs mentioned above, it was unavoidable to include patients with other underlying diseases that may also cause changes in these inflammatory biomarkers. Therefore, besides independent prognostic effects of these inflammatory biomarkers, novel index combining specific inflammatory biomarkers for different cancers (e.g., NLR and LMR for bone sarcomas based on our analysis) to assess their synergistic effects should be further studied as well.

This study had also some limitations that should be clarified. First, the number of studies included in this metaanalysis was not large, and only literature published in English was selected. Second, all included studies were retrospective, observational ones without the data of prospective cohorts. This might have resulted in bias in data analysis. Third, considering that outcomes might vary greatly depending on histological types, we performed subgroup analysis in osteosarcoma, Ewing sarcoma, and chondrosarcoma. Our 
results revealed the prognostic value of NLR in osteosarcoma and Ewing sarcoma, but not in chondrosarcoma. Therefore, studies on specific histological types are still needed to verify our results. Fourth, among all included studies, only 3 provided univariate data, which might have caused potential overestimation of the prognostic role of NLR and LMR. Besides, in the ten studies enrolled for quantitative synthesis, eight of them were from China and it might affect the generalisation of this work. Finally, as heterogeneity was observed among the included studies, we tried to identify the source by performing subgroup analysis. However, the prognostic value of NLR was demonstrated to not be affected in most subgroups, reinforcing the predictive effect of NLR and LMR in the clinical outcomes of patients with bone sarcomas.

\section{Conclusions}

Our meta-analysis demonstrated that higher pretreatment NLR and lower pretreatment LMR were strongly associated with poor prognosis in patients with bone sarcomas. Due to the low cost, high availability, and reproducibility, these inflammatory markers could be used to stratify high-risk patients with bone sarcomas and to provide suitable management strategies. However, to determine the optimal cutoff values of NLR and LMR in the prognosis of bone sarcoma still requires further prospective studies.

\section{Data Availability}

The data used in this paper are available from the corresponding author upon reasonable request.

\section{Conflicts of Interest}

The authors declare that there is no conflict of interest regarding the publication of this paper.

\section{Acknowledgments}

This study was supported by the $\mathrm{PhD}$ research startup foundation of Liaoning Province (No. 20180540024) and 345 Talent Project, Shengjing Hospital of China Medical University. We thank all the authors and participants for their hard work in this study. We appreciate the help of Editage (https://www .editage.cn) for language editing.

\section{References}

[1] J. Y. C. Hui, "Epidemiology and etiology of sarcomas," The Surgical Clinics of North America, vol. 96, no. 5, pp. 901-914, 2016.

[2] D. Reed, Amankwah, and Conley, "Epidemiology and therapies for metastatic sarcoma," Clinical Epidemiology, vol. 5, article 147, 2013.

[3] J. Whelan, A. McTiernan, N. Cooper et al., "Incidence and survival of malignant bone sarcomas in England 1979-2007," International Journal of Cancer, vol. 131, no. 4, pp. E508E517, 2012.
[4] R. L. Siegel, K. D. Miller, and A. Jemal, "Cancer statistics, 2018," CA: A Cancer Journal for Clinicians, vol. 68, no. 1, pp. 7-30, 2018.

[5] D. C. Dean, S. Shen, F. J. Hornicek, and Z. Duan, "From genomics to metabolomics: emerging metastatic biomarkers in osteosarcoma," Cancer Metastasis Reviews, vol. 37, no. 4, pp. 719-731, 2018.

[6] P. A. Thompson and M. Chintagumpala, "Targeted therapy in bone and soft tissue sarcoma in children and adolescents," Current Oncology Reports, vol. 14, no. 2, pp. 197-205, 2012.

[7] J. H. Park, A. J. Fuglestad, A. H. Køstner et al., "Systemic inflammation and outcome in 2295 patients with stage I-III colorectal cancer from Scotland and Norway: first results from the Scotscan Colorectal Cancer Group," Annals of Surgical Oncology, vol. 27, no. 8, pp. 2784-2794, 2020.

[8] M. Papale, M. Buccarelli, C. Mollinari et al., "Hypoxia, inflammation and necrosis as determinants of glioblastoma cancer stem cells progression," International Journal of Molecular Sciences, vol. 21, no. 8, p. 2660, 2020.

[9] D. C. McFarland, D. R. Jutagir, A. H. Miller, W. Breitbart, C. Nelson, and B. Rosenfeld, "Tumor mutation burden and depression in lung cancer: association with inflammation," Journal of the National Comprehensive Cancer Network, vol. 18, no. 4, pp. 434-442, 2020.

[10] J. Wang, Z. Hui, Y. Men et al., "Systemic InflammationImmune Status Predicts Survival in Stage III-N2 Non- Small Cell Lung Cancer," The Annals of Thoracic Surgery, vol. 108, no. 6, pp. 1701-1709, 2019.

[11] A. Stang, "Critical evaluation of the Newcastle-Ottawa scale for the assessment of the quality of nonrandomized studies in meta-analyses," European Journal of Epidemiology, vol. 25, no. 9, pp. 603-605, 2010.

[12] Q. Yang, T. Chen, Z. Yao, and X. Zhang, "Prognostic value of pre-treatment Naples prognostic score (NPS) in patients with osteosarcoma," World Journal of Surgical Oncology, vol. 18, no. 1, p. 24, 2020.

[13] K. Xu, B. Li, Q. Huang et al., "Clinical significance of traditional clinical parameters and inflammatory biomarkers for the prognosis of patients with spinal Chondrosarcoma: a retrospective study of 150 patients in a single center," European Spine Journal, vol. 28, no. 6, pp. 1468-1479, 2019.

[14] X. Huang, H. Hu, W. Zhang, and Z. Shao, "Prognostic value of prognostic nutritional index and systemic immuneinflammation index in patients with osteosarcoma," Journal of Cellular Physiology, vol. 234, no. 10, pp. 18408-18414, 2019.

[15] Y. J. Li, X. Yang, W. B. Zhang, C. Yi, F. Wang, and P. Li, "Clinical implications of six inflammatory biomarkers as prognostic indicators in Ewing sarcoma," Cancer Management and Research, vol. Volume 9, pp. 443-451, 2017.

[16] L. Vasquez, E. León, B. Beltran, I. Maza, M. Oscanoa, and J. Geronimo, "Pretreatment neutrophil-to-lymphocyte ratio and lymphocyte recovery: independent prognostic factors for survival in pediatric sarcomas," Journal of Pediatric Hematology/Oncology, vol. 39, no. 7, pp. 538-546, 2017.

[17] Y. J. Li, K. Yao, M. X. Lu, W. B. Zhang, C. Xiao, and C. Q. Tu, "Prognostic value of the $\mathrm{C}$-reactive protein to albumin ratio: a novel inflammation-based prognostic indicator in osteosarcoma," Onco Targets and Therapy, vol. Volume 10, pp. 5255-5261, 2017.

[18] W. K. Xia, Z. L. Liu, D. Shen, Q. F. Lin, J. Su, and W. D. Mao, "Prognostic performance of pre-treatment NLR and PLR in 
patients suffering from osteosarcoma," World Journal of Surgical Oncology, vol. 14, no. 1, 2016.

[19] B. Liu, Y. Huang, Y. Sun et al., "Prognostic value of inflammation-based scores in patients with osteosarcoma," Scientific Reports, vol. 6, no. 1, article 39862, 2016.

[20] N. Aggerholm-Pedersen, K. Maretty-Kongstad, J. Keller, S. Baerentzen, and A. Safwat, "The prognostic value of serum biomarkers in localized bone sarcoma," Translational Oncology, vol. 9, no. 4, pp. 322-328, 2016.

[21] T. Liu, X. C. Fang, Z. Ding, Z. G. Sun, L. M. Sun, and Y. L. Wang, "Pre-operative lymphocyte-to-monocyte ratio as a predictor of overall survival in patients suffering from osteosarcoma," FEBS Open Bio, vol. 5, no. 1, pp. 682-687, 2015.

[22] Y. Shen, H. Wang, X. Chen, W. Li, and J. Chen, "Prognostic significance of lymphocyte-to-monocyte ratio and plateletto-lymphocyte ratio in patients with hepatocellular carcinoma undergoing transcatheter arterial chemoembolization and radiofrequency ablation," Onco Targets and Therapy, vol. Volume 12, pp. 7129-7137, 2019.

[23] T. W. Kim, J. H. Lee, K. H. Shim et al., "Prognostic significance of preoperative and follow-up neutrophil-to-lymphocyte ratio and platelet-to-lymphocyte ratio in patients with nonmetastatic clear cell renal cell carcinoma," Investigative and Clinical Urology, vol. 60, no. 1, pp. 14-20, 2019.

[24] L. Wang, J. J. Sheng, H. Zhao, L. Su, C. Y. Liu, and S. G. Wan, "The prognostic significance of lymphocyte/monocyte ratio in diffuse large B cell lymphoma," Zhonghua xue ye xue za zhi= Zhonghua Xueyexue Zazhi, vol. 40, no. 5, pp. 436-438, 2019.

[25] K. Furukawa, G. Kawasaki, T. Naruse, and M. Umeda, "Prognostic significance of pretreatment lymphocyte-to-monocyte ratio in patients with tongue cancer," Anticancer Research, vol. 39, no. 1, pp. 405-412, 2019.

[26] M. Ikeda, H. Furukawa, H. Imamura et al., "Poor prognosis associated with thrombocytosis in patients with gastric cancer," Annals of Surgical Oncology, vol. 9, no. 3, pp. 287-291, 2002.

[27] M. McCourt, J. H. Wang, S. Sookhai, and H. P. Redmond, "Proinflammatory mediators stimulate neutrophil-directed angiogenesis," Archives of Surgery, vol. 134, no. 12, pp. 1325$1331,1999$.

[28] M. McCourt, J. H. Wang, S. Sookhai, and H. P. Redmond, "Activated human neutrophils release hepatocyte growth factor/scatter factor," European Journal of Surgical Oncology (EJSO), vol. 27, no. 4, pp. 396-403, 2001.

[29] E. Jabłońska, M. Kiluk, W. Markiewicz, L. Piotrowski, Z. Grabowska, and J. Jabłoński, “TNF-alpha, Il-6 and their soluble receptor serum levels and secretion by neutrophils in cancer patients," Archivum Immunologiae et Therapiae Experimentalis, vol. 49, no. 1, pp. 63-69, 2001.

[30] S. Jaillon, M. R. Galdiero, D. del Prete, M. A. Cassatella, C. Garlanda, and A. Mantovani, "Neutrophils in innate and adaptive immunity," Seminars in Immunopathology, vol. 35, no. 4, pp. 377-394, 2013.

[31] M. Z. Wojtukiewicz, E. Sierko, D. Hempel, S. C. Tucker, and K. V. Honn, "Platelets and cancer angiogenesis nexus," Cancer Metastasis Reviews, vol. 36, no. 2, pp. 249-262, 2017.

[32] S. Jain, J. Harris, and J. Ware, "Platelets: linking hemostasis and cancer," Arteriosclerosis, Thrombosis, and Vascular Biology, vol. 30, no. 12, pp. 2362-2367, 2010.

[33] R. Orellana, S. Kato, R. Erices et al., "Platelets enhance tissue factor protein and metastasis initiating cell markers, and act as chemoattractants increasing the migration of ovarian cancer cells," BMC Cancer, vol. 15, no. 1, p. 290, 2015.

[34] H. Ishii, M. Takahara, T. Nagato et al., "Monocytes enhance cell proliferation and LMP 1 expression of nasal natural killer/T-cell lymphoma cells by cell contact-dependent interaction through membrane-bound IL-15," International Journal of Cancer, vol. 130, no. 1, pp. 48-58, 2012.

[35] M. R. Galdiero, E. Bonavita, I. Barajon, C. Garlanda, A. Mantovani, and S. Jaillon, "Tumor associated macrophages and neutrophils in cancer," Immunobiology, vol. 218, no. 11, pp. 1402-1410, 2013.

[36] T. K. Hoffmann, G. Dworacki, T. Tsukihiro et al., "Spontaneous apoptosis of circulating $\mathrm{T}$ lymphocytes in patients with head and neck cancer and its clinical importance," Clinical Cancer Research, vol. 8, no. 8, pp. 2553-2562, 2002.

[37] H. Li, Q. Su, B. Li et al., "High expression of WTAP leads to poor prognosis of gastric cancer by influencing tumourassociated T lymphocyte infiltration," Journal of Cellular and Molecular Medicine, vol. 24, no. 8, pp. 4452-4465, 2020.

[38] N. al-Rajhi, H. Soudy, S. A. Ahmed et al., "CD3+T-lymphocyte infiltration is an independent prognostic factor for advanced nasopharyngeal carcinoma," BMC Cancer, vol. 20, no. 1, p. 240, 2020.

[39] X. Xie, S. Gong, H. Jin et al., "Radiation-induced lymphopenia correlates with survival in nasopharyngeal carcinoma: impact of treatment modality and the baseline lymphocyte count," Radiation Oncology, vol. 15, no. 1, p. 65, 2020.

[40] M. Warny, J. Helby, B. G. Nordestgaard, H. Birgens, and S. E. Bojesen, "Incidental lymphopenia and mortality: a prospective cohort study," Canadian Medical Association Journal, vol. 192, no. 2, pp. E25-E33, 2020.

[41] Y. Li, X. Liu, J. Zhang, and W. Yao, "Prognostic role of elevated preoperative systemic inflammatory markers in localized soft tissue sarcoma," Cancer Biomarkers, vol. 16, no. 3, pp. 333342, 2016.

[42] Y. Liang, W. Wang, Y. Que et al., "Prognostic value of the fibrinogen/albumin ratio (FAR) in patients with operable soft tissue sarcoma," BMC Cancer, vol. 18, no. 1, article 942, 2018.

[43] G. Liu, L. C. Ke, and S. R. Sun, "Prognostic value of pretreatment neutrophil-to-lymphocyte ratio in patients with soft tissue sarcoma: a meta-analysis," Medicine, vol. 97, no. 36, article e12176, 2018.

[44] L. Q. Li, Z. H. Bai, L. H. Zhang et al., "Meta-analysis of hematological biomarkers as reliable indicators of soft tissue sarcoma prognosis," Frontiers in Oncology, vol. 10, p. 30, 2020.

[45] X. Li, F. Tian, F. Wang, and Y. Li, "Serum C-reactive protein and overall survival of patients with osteosarcoma," Tumor Biology, vol. 36, no. 7, pp. 5663-5666, 2015.

[46] X. Su, J. Teng, G. Jin et al., "ELK1-induced upregulation of long non-coding RNA MIR100HG predicts poor prognosis and promotes the progression of osteosarcoma by epigenetically silencing LATS1 and LATS2," Biomedicine \& Pharmacotherapy, vol. 109, pp. 788-797, 2019.

[47] M. Liu, P. Yang, G. Mao et al., "Long non-coding RNA MALAT1 as a valuable biomarker for prognosis in osteosarcoma: A systematic review and meta-analysis," International Journal of Surgery, vol. 72, pp. 206-213, 2019. 